\title{
Viabilidade do cultivo de pinhão-manso na bacia do Rio Piquiri no município de Palotina-PR ${ }^{1}$
}

\author{
Kenia Gabriela dos Santos ${ }^{2}$, Elisandro Pires Frigo ${ }^{3}$, Caroline Thaís Eckert ${ }^{4}$, Thiago \\ Stock Paschoal ${ }^{5}$, Roberto Luiz de Carli $^{6}$, Rafaela Koglin Bastos ${ }^{7}$
}

\footnotetext{
${ }^{1}$ Aceito para publicação em $2^{\circ}$ trimestre de 2014

${ }^{2}$ Mestranda em Energia na Agricultura, Universidade Estadual do Oeste do Paraná;

${ }^{3}$ Prof. Dr. Adjunto da Universidade Federal do Paraná UFPR- Setor Palotina/PR, Brasil.

${ }^{4}$ Mestranda em Energia na Agricultura, Universidade Estadual do Oeste do Paraná;

${ }^{5}$ Programa de Pós-Graduação em Energia na Agricultura, Universidade Estadual do Oeste do Paraná;

${ }^{6}$ Programa de Pós-Graduação em Energia na Agricultura, Universidade Estadual do Oeste do Paraná;

${ }^{7}$ Mestranda em Energia na Agricultura, Universidade Estadual do Oeste do Paraná.
}

\section{Resumo}

As bacias hidrográficas possuem aspectos físicos influenciadores no seu comportamento hidrológico. Desta forma, este trabalho objetiva analisar a morfometria de um segmento da bacia hidrográfica do Rio Piquiri, localizada no município de Palotina. Em seguida, realizar o zoneamento climático para a região de estudo, com o intuito de definir a viabilidade para o cultivo de pinhão-manso e a distância média de transporte (DMT) deste para as usinas de biodiesel do Paraná. A partir da análise dos dados foi possível concluir que a área corresponde a $378,506 \mathrm{~km}^{2}$, fator de forma 0,25 , coeficiente de compacidade 1,10, índice de conformação 0,39, declividade média da área da bacia de 333,86 metros, declividade do curso d'água $0,00585 \mathrm{~m} \cdot \mathrm{m}^{-1}\left(\mathrm{~S}_{1}\right), 0,00543 \mathrm{~m} \cdot \mathrm{m}^{-1}\left(\mathrm{~S}_{2}\right)$ e $0,0047 \mathrm{~m} \cdot \mathrm{m}^{-1}\left(\mathrm{~S}_{3}\right)$, densidade do curso d'água $0,00449 \mathrm{~km}$ e de drenagem $0,384 \mathrm{~km} / \mathrm{km}^{2}$.Considerando-se as características desta área este trabalho conclui que o município estudado revela-se apto para o cultivo de pinhão-manso em relação às necessidades térmicas e hídricas. E a DMT é mais favorável dentro do estado do Paraná são para as cidades de Céu Azul, Santa Helena e Tuneiras do Oeste, onde a distância total a ser percorrida não é maior que 250 $\mathrm{Km}$.

Palavras-chave: Caracterização, biodiesel, Jatropha curcas 


\title{
Viability of cultivation of Jatropha curcas in Piquiri River basin in Palotina-PR ${ }^{1}$
}

\begin{abstract}
Watersheds have influencers physical aspects in their hydrological behavior. Thus, this work aims to analyze the morphology of a segment of the river basin Piquiri located in Palotina. Then perform the climatic zoning for the area of study, in order to define the feasibility for growing jatropha and the average transport distance (ATD) for this biodiesel plants of Paraná. From the data analysis it was concluded that the area corresponds to $378.506 \mathrm{~km}^{2}$, so 0.25 factor, compactness coefficient of $1.10,0.39$ index conformation, average slope of the basin area of 333.86 meters, slope of the watercourse $0.00585 \mathrm{~mm}^{-1}\left(\mathrm{~S}_{1}\right), 0.00543 \mathrm{~mm}^{-1}\left(\mathrm{~S}_{2}\right)$ and $0.0047 \mathrm{~mm}^{-1}\left(\mathrm{~S}_{3}\right)$, density of $0.00449 \mathrm{~km}$ watercourse and drainage $0.384 \mathrm{~km} / \mathrm{km}^{2}$. Considerando the characteristics of this area this paper concludes that the city studied proves to be suitable for the cultivation of jatropha in relation to temperature and water requirements. And ATD is more favorable within the state of Paraná are for the cities of Blue Heaven, Helena and Tuneiras West, where the total distance to be traveled is not greater than $250 \mathrm{Km}$.
\end{abstract}

Key-words: Characterization, biodiesel, Jatropha curcas

\section{Introdução}

A ocupação e uso do solo por atividades agrícolas alteram sensivelmente os fatores biológicos, físicos e químicos dos sistemas naturais. Essas alterações ocorridas em uma bacia hidrográfica podem ser estudadas através do monitoramento da qualidade da água (MERTEN et al., 2002; SILVA et al., 2010).

Pode-se definir bacia hidrográfica como: área composta por uma rede de cursos d'água, de captação natural de água da precipitação, irá convergir a maioria do escoamento a um ponto de menor declividade, exutório, que posteriormente abastecerá o lençol freático (TONELLO et al., 2006; FIRDAUS et al., 2014).

A qualidade e a disponibilidade de recursos hídricos estão relacionadas aos problemas em sua conservação. Para compreensão de fatores que influenciam, nesta conservação, em uma bacia hidrográfica torna-se essencial o conhecimento de suas características morfométricas (SANTOS et al., 2012). 
Sendo assim, as características principais são: a área de drenagem, o comprimento e declividade da bacia. A análise morfométrica dessas características hidrográficas consiste no levantamento de índices e valores numéricos de vários atributos da bacia, permitindo a compreensão do funcionamento do sistema de drenagem. As modificações morfológicas da bacia evoluem de acordo com a ocorrência da combinação dos fatores naturais e antrópicos (LLORME; GRIFFIS, 2013; SAMARAS; KOUTITAS, 2014).

Segundo Souza et al. (2012) uma das análises importantes e iniciais de uma bacia hidrográfica é a caracterização hidrológica deste local, pois, expõe informações importantes sobre o comportamento hidrológico desta. Além disso, a vulnerabilidade ambiental de uma bacia pode ser indicada por estas análises.

Os aspectos físicos e bióticos das bacias hidrográficas desempenham importante atividade no seu comportamento hidrológico, tornando importante o acompanhamento numérico do sistema, usando dados que avaliem as culturas atuais da bacia, que com o aproveitamento da sua tipologia hidrológica, aumentam em seu rendimento (ALVEZ et al., 2013).

Neste sentido, locais com déficits hídricos promovem perdas na produtividade de algumas culturas como milho, soja, algodão, entre outras. Isto ocorre devido a exigência hídrica e térmica para que estas se desenvolvam (FARIA, 2013) enquanto outras culturas são menos exigentes. Com isto, o pinhão-manso destaca-se, pois é considerado propício para locais com baixa disponibilidade nestes dois aspectos (BARBOZA, 2011).

O pinhão-manso é uma espécie pouco exigente quanto a condições climáticas e fertilidade do solo, possuindo grande adaptabilidade em distintas regiões, no entanto, não tolera geadas agressivas, mas sobrevive a geadas menos consideráveis, que gera senescência em suas folhas, reduzindo em sua produção (JINGURA et al., 2011). O cultivo de pinhão-manso é recente, contendo limitações técnicas e pode ocasionar resultados não satisfatórios para o setor agrícola e industrial (SINGH et al., 2014).

Além das condições requeridas para o seu desenvolvimento, esta cultura destacase pela presença de óleo em suas sementes que o destina para diversas finalidades, como a produção de sabões, lubrificantes, tintas e biodiesel. Este último é constituído por ésteres alquílicos de ácidos carboxílicos de cadeia longa, e a matéria-prima comumente utilizada são óleos vegetais, com isto, o uso do óleo do pinhão-manso para a síntese deste 
biocombustível tem sido impulsionado por inúmeras pesquisas na área (PEIXOTO, 1973; PEDROSO, et al. 2010; RAO et al., 2012).

Desta forma, este trabalho tem como o objetivo delimitar e caracterizar a bacia (sua área, fator de forma, coeficiente de compacidade, índice de conformação, declividade da bacia hidrográfica e do curso d'água, densidade do curso d'água e de drenagem). Em seguida, verificar a possibilidade de implantação da cultura do pinhão-manso na área, presumindo o desenvolvimento ou não da cultura, com relação aos aspectos hídricos, térmicos e solo, e a viabilidade com relação DTM para o transporte do pinhão-manso cultivado até as usinas de biodiesel no Estado do Paraná.

\section{Material e métodos}

Área de estudo

A área em estudo está situada na bacia hidrográfica do Rio Piquiri, no município de Palotina com coordenadas latitude $24^{\circ} 17^{\prime} 02^{\prime \prime}$ e longitude $53^{\circ} 50^{\prime} 24^{\prime}$ '. Os solos da região são predominantemente argilosos, com coloração vermelho forte e sendo uma provável transição de nitossolo e latossolo eutrófico, na superfície aparentemente é um solo seco.

A região é caracterizada com atividades agropecuárias, dependendo diretamente das condições climáticas. De acordo com a classificação climática de Köppen-Geiger na região há a predominância do clima subtropical, do tipo Cfa, e as temperaturas médias para meses frios e quentes correspondem a $18^{\circ} \mathrm{C}$ e $22^{\circ} \mathrm{C}$, respectivamente (Pedron et al., 2013).

A bacia hidrográfica localizada na região foi delimitada e caracterizada (Tabela 1) com o objetivo de obter informações que auxiliarão na compreensão dos estudos hidrológicos desta, e a verificar se é possível realizar o cultivo do pinhão-manso na área. A caracterização da bacia hidrográfica baseou-se na utilização de programas como Auto Cad e Excel.

Tabela 1. Características morfométricas obtidas no estudo da bacia hidrográfica da região de Palotina

\begin{tabular}{ll}
\hline Características físicas & Coeficiente de compacidade (Eq. 1) \\
& Fator de forma (Eq. 2) \\
& Índice de conformação (Eq. 3) \\
\cline { 1 - 2 } Características do relevo & Declividade da bacia hidrográfica \\
\cline { 1 - 2 } &
\end{tabular}

Revista Brasileira de Energias Renováveis, v. 3, p. 136-150, 2014 


\begin{tabular}{ll}
\hline & $\begin{array}{l}\text { Declividade do curso d'água } \\
\text { (Eq.4, 5, 6) }\end{array}$ \\
\hline Características drenagem & $\begin{array}{l}\text { Densidade do curso d'água (Eq. 7) } \\
\text { Densidade de drenagem (Eq. 8) }\end{array}$ \\
\hline
\end{tabular}

$K c=0,28 \frac{P}{\sqrt{A}}$

$F f=\frac{B}{L}$

$F c=\frac{A}{L^{2}}$

Em que:

$\mathrm{P}=$ perímetro da bacia em $\mathrm{km}$

$\mathrm{A}=$ área da bacia em $\mathrm{km}^{2}$

$\mathrm{L}=$ comprimento da bacia

$\mathrm{B}=$ largura média da bacia

$S_{1}=\frac{\Delta H}{L}$

(Eq. 4)

$S_{2}=\frac{2 \times A_{b_{p}}}{L^{2}}$

$S_{3}=\left(\frac{L}{\sum_{1}^{n} \frac{L i}{\sqrt{I i}}}\right)$

Em que:

$\Delta \mathrm{H}=$ variação da cota entre dois pontos externos

$\mathrm{L}=$ comprimento do rio

$\mathrm{Apb}=$ área abaixo do perfil

$\mathrm{Li}$ = extensão horizontal de cada um dos $\mathrm{n}$ trechos

$\mathrm{Ii}=$ declividade de cada um dos $\mathrm{n}$ trechos $(\mathrm{Ii}=\mathrm{Hi} / \mathrm{Li})$ 


$$
\begin{aligned}
& D s=\frac{N_{s}}{A} \\
& D d=\frac{L}{A}
\end{aligned}
$$

Em que:

Ns = número de cursos d'água

$\mathrm{L}=$ comprimento total dos cursos d'água

\section{Determinação meteorológica do munícipio de Palotina/PR}

Os dados históricos meteorológicos foram disponibilizados pelos institutos: IAPAR (Instituto Agronômico do Paraná), COPEL (Companhia Paranaense de Energia), ÁGUAS PARANÁ (Instituto das Águas do Paraná) com duas estações meteorológicas na localidade e SIMEPAR (Sistema de Informações Meteorológicas do Paraná).

Caracterização do solo

Os dados das características do solo foram obtidos por meio das informações da Empresa Brasileira de Pesquisa Agropecuária (Embrapa, 1999).

\section{Implantação do cultivo de pinhão-manso na área da bacia caracterizada}

Para o cultivo de pinhão-manso deve-se considerar as condições do local, pois, fatores como fertilidade do solo, climáticas, condições de mercado e logística influenciam na implantação da cultura. Com isto, após a definição do tipo de solo, condições hídricas e térmicas do local, determinou-se as partes viáveis da bacia para a inserção do pinhãomanso com relação a declividade requerida por este, e a viabilidade com relação a DMT para o escoamento da produção de pinhão-manso às usinas de biodiesel do Paraná.

\section{Resultados e discussão}

Caracterização da bacia

A área e o perímetro da área da bacia do Rio Piquiri correspondem a 378,506 $\mathrm{km}^{2} \mathrm{e} 76,861 \mathrm{~km}$, respectivamente. Estes valores foram obtidos por meio do uso do Auto Cad com base nas curvas de níveis (Figura 1). Na Tabela 2 encontra-se os resultados da caracterização da área da bacia hidrográfica do Rio Piquiri. 




Figura 1. Bacia hidrográfica e suas curvas de nível

A área total analisada é caracterizada com:

- $\quad$ Área de floresta (APP, Reservas, Parques, etc.) $3.100 .000,00 \mathrm{~m}^{2}$ equivalente a aproximadamente $8,19 \%$.

- Urbano (Edificações, Rodovias, Cidades, etc.) 5.590.000,12 $\mathrm{m}^{2}$ equivalente a aproximadamente $1,47 \%$.

- Estradas rurais $690.000,00 \mathrm{~m}^{2}$ equivalente a aproximadamente $0,18 \%$.

- $\quad$ Terra utilizada (Lavouras, Pastagens, etc.) $369.120 .000,00 \mathrm{~m}^{2}$ equivalente a aproximadamente $90,16 \%$.

Tabela 2. Características da bacia hidrográfica

\begin{tabular}{cc}
\hline $\begin{array}{c}\text { Características } \\
\text { morfométricas }\end{array}$ & Resultados \\
\hline Coeficiente de compacidade & 1,10 \\
Fator de forma & 0,25 \\
Índice de conformação & 0,39 \\
Declividade da bacia & 333,86 \\
hidrográfica $(\mathrm{m})$ &
\end{tabular}




$\begin{array}{cc}\text { Declividade do curso d'água } & \mathrm{S}_{1} 0,00585 \\ \left(\mathrm{~m} \cdot \mathrm{m}^{-1}\right) & \mathrm{S}_{2} 0,00543 \\ & \mathrm{~S}_{3} 0,0047 \\ \text { Densidade do curso d'água } & 0,0449 \\ \left(\mathrm{~km} / \mathrm{km}^{2}\right) & \\ \text { Densidade de drenagem } & 0,384 \\ \left(\mathrm{~km} / \mathrm{km}^{2}\right) & \end{array}$

Com valores dos parâmetros determinados na bacia (Tabela 2) obtêm-se que esta possui um coeficiente de compacidade correspondente a 1,10 e fator de forma 0,25. Segundo Tucci (1993) quando o valor desse coeficiente de compacidade é superior a 1 a bacia é caracterizada com um formato alongado, e quanto maior este índice mais irregular é seu formato. $\mathrm{O}$ índice de conformação é de 0,39 consequentemente induz a um formato alongado. Esses valores revelam um local com menor risco de enchentes (VILLELA; MATTOS, 1975).

Nesta área a declividade média corresponde a 333,86 m, e quanto menor este valor menor é a velocidade de escoamento, consequentemente maior é o tempo de concentração da água no local. Esta análise foi determinada pela relação das curvas de níveis, e é considerada um parâmetro influenciador no escoamento superficial do local, além disso, há relação com a presença de erosão das proximidades, promovendo a perda do solo, nutrientes e matéria-orgânica (TONELLO, 2006).

$\mathrm{Na}$ declividade do curso d'água, os valores encontrados para $\mathrm{S}_{1}, \mathrm{~S}_{2}$ e $\mathrm{S}_{3}$ foram de $0,00585,0,00543$ e $0,0047 \mathrm{~m} . \mathrm{m}^{-1}$, respectivamente, os quais indicam pequenas variações da declividade do percurso do canal principal. Com relação ao comportamento hidrológico, este é considerado baixo caracterizado com densidade do curso d'água principal de $0,00449 \mathrm{~km}$ e baixa densidade de drenagem $0,384 \mathrm{~km} / \mathrm{km}^{2}$.

De acordo com Christofoletti (1980) a densidade do rio é muito importante, uma vez que esta permite identificar o comportamento da área, sendo que um valor reduzido indica menor capacidade de geração de cursos d'água. O resultado obtido na caracterização deste parâmetro mostra que a área possui uma densidade de rios baixa quando comparada com a área total da bacia.

A área também apresenta baixa capacidade de drenagem $\left(0,384 \mathrm{~km} / \mathrm{km}^{2}\right)$. As bacias com drenagem superior a $3,5 \mathrm{~km} / \mathrm{km}^{2}$ possuem alta capacidade de drenagem, isto 
é devido a alta concentração de precipitação. Além disso, esta área da bacia é caracterizada como de quarta ordem, não sendo muito ramificada.

Tipo de solo da bacia

A bacia caracteriza-se com o solo do tipo LVe -- Latossolo Vermelho Eutrófico de textura muito argilosa $(>60 \%)($ Embrapa, 1999). Onde:

$1^{\circ}$ nível - Latossolo - L

$2^{\circ}$ nível - Vermelho - V

$3^{\circ}$ nível - eutrófico - e

O solo apresenta granulometria bem graduada, com cerca de $60 \%$ de argila, $19 \%$ de silte e $21 \%$ de areia (Embrapa, 2006). O pinhão-manso mostra-se favorável ao solo desta área da bacia, pois de acordo com Ye et al. (2009) esta oleaginosa cresce facilmente em solos pedregosos e de baixa umidade. Além disso, a produtividade de frutos e óleo é maior em solos férteis com boas características físicas e irrigação (Tikkoo et al., 2013), como é o caso desta bacia.

$\mathrm{O}$ crescimento do pinhão-manso é limitado em declividades superiores a $30^{\circ}$. A bacia caracterizada apresentou declividades inferiores a $30^{\circ} \mathrm{C}$ (Figura 3), as quais favorecem o cultivo dessa oleaginosa no local.



Figura 3. Declividade da bacia 
De acordo com Bastos (2013) o cultivo do pinhão-manso no munícipio de Palotina tratado com água residuária de suinucultura apresentou baixo índice de acidez do óleo, tornando-o viável para a produção de biodiesel.

\section{Situação meteorológica do município de Palotina}

De acordo com os dados expostos na Figura 4, o volume de precipitado foi consideravelmente heterogêneo nos últimos 42 anos, em destaque o ano de 1983 com médias de $230 \mathrm{~mm}$ mensais, sendo que posteriormente houve um decréscimo, no ano de 1985, com média de 87 mm mensais.

Para a cultura do pinhão-manso considera-se ideal o índice pluviométrico de, em média, 150 mm mensais (DUKE, 1983), sendo observado em comparação com os dados históricos do local.



Figura 4. Precipitação média anual de Palotina 1965-2007

Os dados climatológicos de temperaturas médias de Palotina, foram obtidos pelo SIMEPAR. As temperaturas mínimas foram heterogêneas, sendo que o ano de 2000 destacou-se por ter as maiores temperaturas desse índice, com $28{ }^{\circ} \mathrm{C}$, e o período de menores temperaturas foi em 1976, com uma média mensal de $15^{\circ} \mathrm{C}$.

Em contraste com os dados mínimos, os índices máximos foram mais homogêneos, no entanto, se destacou o ano de 2002 com altas temperaturas, $30{ }^{\circ} \mathrm{C}$ mensais, e o ano de 1983 obteve a marca de menores temperaturas para o índice de temperatura máxima do ar mensal, com $27^{\circ} \mathrm{C}$, como pode ser visualizado na Figura 5 , abaixo.

De acordo com Heller (1983) e Dallacort et al. (2010), a Jatropha curcas possui bom desenvolvimento em áreas tropicais, que mantém uma temperatura média de $24{ }^{\circ} \mathrm{C}$. 
Do contrário, baixas temperaturas podem causar a senescência da planta, causando queda foliar e diminuição da produção de sementes.



Figura 5. Histórico médio de temperaturas de Palotina 1973-2003

\section{DMT do pinhão-manso}

A distância a ser percorrida do ponto médio (centro da bacia) até o centro da cidade de Palotina, utilizando o trajeto através de estrada rural e BR, a DMT corresponde a $62 \mathrm{Km}$.

A partir do perímetro urbano, foi elaborado uma malha/rede de distribuição até as usinas beneficiadoras de biodiesel. Conforme os dados da ANP - Agencia Nacional do Petróleo (2008), foram identificadas no Estado do Paraná 21 Usinas, sendo que destas 19 em funcionamento e outras 3 em fase de projeto/execução.

Com base nestes dados da ANP (2008) foram estabelecidas rotas para a distribuição da produção do pinhão-manso na área da bacia do Rio Piquiri, tendo como ponto de referência para estabelecer a DMT a cidade de Palotina. Na Tabela 3 encontrase o raio de distribuição do pinhão-manso.

Tabela 3. Rede de Distribuição do Pinhão-manso

\begin{tabular}{cc}
\hline Cidade de destino & $\begin{array}{c}\text { Distância em Km a partir de Palotina - } \\
\text { DMT }\end{array}$ \\
\hline Andirá & 468 \\
Araucária* & 587 \\
Campo Largo & 567 \\
Céu Azul & 120 \\
Curitiba & 593
\end{tabular}




\begin{tabular}{cc} 
Ivaiporã & 316 \\
Londrina* & 351 \\
Marialva & 271 \\
Ponta Grossa* & 501 \\
Rolândia & 330 \\
Roncado & 219 \\
Santa Helena & 104 \\
Tuneiras do Oeste & 162 \\
\hline
\end{tabular}

*Cidades com mais de uma usina instalada.

Fonte: ANP - Agência Nacional do Petroleo (2008)

Com base nos dados apresentados, observa se que para o transporte da produção do pinhão-manso, para o seu beneficiamento e transformação em biodiesel, tornando-se indispensável a avaliação logística, com relação a sua DMT, deve ser escoada para as cidades de Céu Azul, Santa Helena e Tuneiras do Oeste, onde a distância total a ser percorrida não é maior que $250 \mathrm{Km}$.

\section{Conclusão}

A bacia possui um formato alongado e baixo comportamento hidrológico, com menor capacidade de geração de cursos d'água, e a região apresenta baixa precipitação.

O segmento da bacia do Rio Piquiri revela-se apto ao cultivo de pinhão-manso, pois, todos os parâmetros descritos neste trabalho como: tipo de solo, declividade, condições hídricas e térmica são favoráveis ao cultivo. Além disso, a DMT da oleaginosa é mais favorável para as cidades de Céu Azul, Santa Helena e Tuneiras do Oeste, onde a distância total a ser percorrida não é maior que $250 \mathrm{Km}$. 


\section{Referências Bibliográficas}

ANP - AGÊNCIA NACIONAL DO PETRÓLEO. Usinas de biodiesel no Brasil. Disponível em: < http://usinasbr.blogspot.com.br/2008/04/paran-18.html> Acesso em: $16 / 06 / 2014$.

ALVEZ, A. R., FRIGO, E. P., SANTOS, C. A., FORNASARI, C. H., MARI JUNIOR, A., MARI, A. G. Viabilidade para o cultivo de soja devido as relações térmicas e hídricas na Bacia do Rio Ipiranga no Município de Santa Terezinha do Itaipu. v. 4, p. 45-62, 2013.

BARBOZA, C.R.A.J Desenvolvimento de mudas de pinhão-manso (Jatropha curcas L.) em tubetes e submetidas a diferentes níveis de déficit hídrico. Tese. 95p. Doutorado em Ciências. Universidade de São Paulo, Escola Superior de Agricultura, São Paulo, 2011.

BASTOS, R.K. Influência da água residuária da suinocultura sobre a acidez no óleo do pinhão-manso (Jatropha curcas L.). Trabalho de Conclusão de Curso. Tecnologia em Biocombustíveis. Universidade Federal do Paraná, 2013.

CHRISTOFOLETTI, A. Geomorfologia. 2 ed. São Paulo: Edgard Blücher, 1980, 180p.

DALLACORT, R.; MARTINS, J.A.; INOUE, M.H.; FREITAS, P.S.L.; KRAUSE, W. Aptidão agroclimática do pinhão-manso na região de Tangará da Serra, MT. Revista Ciênca Agronômica, v. 41, n.3, p. 373-379, 2010..

DUKE, J. A., Handbook of Energy Crops. 1983. Disponível em < https://www.hort.purdue.edu/newcrop/duke_energy/Jatropha_curcas.html> Acesso: 20/06/2014.

EMBRAPA - Empresa brasileira de pesquisa agropecuária. Sistema Brasileiro de Classificação de Solo. Rio de Janeiro: 2. ed, 2006.

EMBRAPA, - Empresa brasileira de pesquisa agropecuária. Sistema Brasileiro de Classificação de solos. Brasília: 1. ed, 1999.

FARIA, C.A.J. Adaptabilidade da cultura do crambe no estado de Mato Grosso. Dissertação de Mestrado. Programa de Pós-Graduação em Ambiente e Sistemas de Produção Agrícola. 67p. Universidade do Estado do Mato Grosso, 2013.

FIRDAUS, R.; NAKAGOSHI, N.; IDRIS, A. sustainability assessment of humid tropical watershed: a case of Batang Merao watershed, Indonesia. Procedia Environmental Sciences, v. 20, p. 722-731, 2014.

HELLER, J. Physic Nut: Jatropha curcas. 1996. Disponível em: <http://www.bionica.info/biblioteca/Heller1996Jatropha.pdf> Acesso: 20/06/2014.

ILORME, F.; GRIFFIS, V.W. A novel procedure for delineation of hydrologically homogeneous regions and the classification of ungauged sites for design flood estimation. Journal of Hydrology, v. 492, p. 151-162, 2013. 
JINGURA, R.M.; MATENGAIFA, R.; MUSADEMBA, D.; MUSIYIWA, K. Characterisation of land types and agro-ecological conditions for production of Jatropha as a feedstock for biofuels in Zimbabwe. Biomass and Bioenergy, v. 35, n.5, p. 2080-2086, 2011.

MERTEN, G. H., MINELLA, J. P. Qualidade da água em bacias hidrográficas rurais: um desafio atual para a sobrevivência futura. Revista de Agroecologia e Desenvolvimento. Rural. Sustentável. Porto Alegre, v.3, n.4, out/dez 2002.

PEDRON, T.P.; MARIANI, K.L.; FARIAS, J.L.R.; ROSSETTO, A. Comportamento do clima de Palotina/PR de 1973 a 2010. Scientia Agraria Paranaensis - SAP, v.12, p. 411-419, 2013.

PEDROSO, L. R. M; DERSCH, P. M; CAVALCANTI, E. H. S; BARRETO, A. J. B; FERREIRA, J. L. P; MOURA, B, S; MENDES, F; PINTO, C. L. S. Extração e caracterização do óleo do pinhão-manso obtido a partir de plantio em Resende - RJ. In: $4^{\circ}$ CONGRESSO DA REDE BRASILEIRA DE TECNOLOGIA DE BIODIESEL, $7^{\circ}$ CONGRESSO BRASILEIRO DE PLANTAS E OLEAGINOSAS, ÓLEOS, GORDURAS E BIODIESEL, Belo Horizonte, 2010. v. 2. p. 675.

PEIXOTO, A. R. Plantas oleaginosas arbóreas. São Paulo, Nobel, 1973. p. 283.

RAO, A.V.R.K.; WANI, S.P.; SINGH, P.; SRINIVAS, K.; RAO, C.S. Water requirement and use by Jatropha curcas in a semi-arid tropical location. Biomass and Bioenergy, $v$. 39, p. 175-181, 2012.

SAMARAS, A.G.; KOUTITAS, C. G. The impact of watershed management on coastal morphology: A case study using an integrated approach and numerical modeling. Geomorphology, v. 211, p. 52-63, 2014.

SANTOS, D.B.; VIDOTTO, M.L.; BERTINATTO, R.; MARCON, G.R.S.; FRIGO, E.P. Caracterização morfométrica da bacia hidrográfica do rio São José, Cascavel, PR. Revista Brasileira de Tecnologia Aplicada nas Ciências Agrárias, v. 5, n. 2, p. 7-18, 2012.

SILVA, D.F.; GALVÍNCIO, J.D.; ALMEIDA, H.R.R.C. Variabilidade da qualidade de água na bacia hidrográfica do rio são francisco e atividades antrópicas relacionadas. Qualitas, v.9, n.3, p. 1-17, 2010.

SINGH, K.; SINGH, B.; VERMA, S.K.; PATRA, D.D. Jatropha curcas: A ten year story from hope to despair. Renewable and Sustainable Energy Reviews, v. 35, p. 356-360, 2014.

SOUZA, A.C.M.; SILVA, M.R.F.; DIAS, N.S. Gestão de recursos hídricos: o caso da bacia hidrográfica Apodi/Mossoró (RN). IRRIGA Brazilian Journal of Irrigation and Drainage, v. 1, n. 1, p. 280-296, 2012.

TIKKOO, A.; YADAV, S.S.; KAUSHIK, N. Effect of irrigation, nitrogen and potassium on seed yield and oil content of Jatropha curcas in coarse textured soils of northwest India. Soil and Tillage Research, v. 134, p. 142-146, 2013. 
TONELLO, K. C., DIAS, H. C. T., DE SOUZA, A. L., RIBEIRO, C. A. A. S., LEITE, F. P. Morfometria da Bacia Hidrográfica da Cachoeira das Pombas, Guanhães - MG. R. Árvore, Viçosa-MG, v.30, n.5, p.849-857, 2006.

TUCCI, C. E. M. Hidrologia, ciência e aplicação. Coleção ABRH de Recursos Hídricos. Vol. 4. Porto Alegre: EDUSP/ABRH, 943p., 1993.

VILLELA S.M.; MATTOS, A. Hidrologia Aplicada. São Paulo: McGraw-Hill do Brasil, 245 f., 1975.

YE, M.; LI, C.; FRANCIS, G.; MAKKAR, H.P.S. Current situation and prospects of Jatropha curcas as a multipurpouse tree in China. Agroforestry Suystems. Dordrecht v. 76, p. 487-497. 2009. 